\title{
Effects of Technical Imagery Training on Neurotransmitters in the Brain of Throwing Curling Stage
}

\author{
Guo $\mathrm{Lu}^{1, \mathrm{a}}$, Dan $\mathrm{Li}^{2, \text { a }}$ and Xiaofang Cui ${ }^{3, \mathrm{a}}$ \\ ${ }^{1}$ Xincheng Street 2888, Jilin Agricultural University, Changchun, Jilin Province 130118, PR China, \\ Department of Sports and Teaching, Jilin Agricultural University \\ ${ }^{2}$ College of sports humanities and Social Sciences, Jilin Institute of Physical Education, Jilin \\ Province 130118,PR China \\ a360282476@qq.com
}

Keywords: EFG; Yoga; Meditation and appearance; Neurotransmitter

\begin{abstract}
Through the 6 months of yoga core technology appearance training to understand the curling athletes in the preparation of curling throwing brain neurotransmitter changes in the situation. The main use of the experimental method, draw them in the preparation stage there is no difference in gender Touhu, only in the S1 (GABA), S2 (GA), S3 (Ach receptor), 5-HT, NE, DA these 6 neurotransmitters have differences, and the core technology of imagery training in different gender before and after test, S2GA (glutamic acid) showed a rising trend;S3 (Ach receptor), 5-HT (serotonin) and DA (dopamine) showed a decrease. Curling athletes in the preparation of curling thrown in the brain within the neurotransmitter is a change in the future can be further studied in this regard.
\end{abstract}

\section{Preface}

In recent years, China's competitive sports has made remarkable achievements, while the ice and snow sports project has shown an indispensable position, curling movement is an intelligent and technical projects, with the project characteristics, its development in China Has a greater advantage and development potential. [1]In the curling technique, the pot is put on the curling with the appropriate force and rotation through the pre-set route to the designated target point. As the pot is the most basic and curving movement is one of the most complex technology [2], the quality of the pot can basically determine the level of the outcome of the game. [3]Cast pot success rate is to evaluate the curling team play a valid indicator, is the key to the success of the game. [4] the actual cast pot stage should be prepared from the pot, began to vote pot, taxi and shot four stages. [5]After the curling shot, throw pot athletes can no longer directly control the curling, showing the pot in the preparatory stage may have been designed to vote pot strategy and route. For the Chinese curling movement, to improve the accuracy of throwing is the current competitive curling training in China must seriously face and properly solve the problem. [6] The core technical representation training is mainly through the specific special movement of the technical points, the use of athletes on the game or training real scene realistic imagination core cast pot technical action to achieve its technical point of automation.2012 Northeast Normal University graduate students Yin Gaabao has confirmed that "tennis players through the quiet EEG alpha wave index changes, and the elephant (the real scene of the game or training realistic imagination called the elephant) EEG and neurotransmission qualitative change.From the quiet to the memory of the brain when the typical neurotransmitters have changed, have significant differences, the individual has a high degree of significant differences. "There are "5- HT in a quiet, mainly on the right side of the brain is active, dopamine is mainly active in the brain, but in the memory like state 5- serotonin and dopamine are active in reverse, and jointly safeguard the neural activity of brain"It can be seen that Yin Jiabao (2012) described the image and this article to illustrate the concept of consistent training is the concept of the study of the cast pot of the core technical appearance training may also improve the curling athletes brain neurotransmitter changes. How can the depth of the brain of the athlete or his or her lovers raise the depths of the brain and ask the researcher questions? And the curling athlete 
is ready to vote when the core technical appearance of the training presented by the neurochemical neurotransmitters, to explore the yoga core technology appearance training on the pot preparation period of brain neurotransmitter changes, in order to improve the preparation and stability of the pot, Sports provides scientific training methods.

\section{Experimental Objects and Methods}

Experimental Object. National Youth Curling Team athletes (male 19, female 8) for a period of 6 months of experimental testing. Subjects were healthy, without any brain-related illnesses. The age is $19.79 \pm 3.79$ years for boys and $20.23 \pm 1.29$ for girls. The training period is $10.37 \pm 1.19$ years. The best result of male team is the fourth in the 2014 Sochi Olympic Games.

Experimental Method. (1) Experimental Tools. Shenzhen cornley High Technology Company, the company developed the SP03 electroencephalograph (International 10/20 system electrode placement method,The use of Professor Mei Lei (1984) the central neurotransmitter system development of ultra slow fluctuations on the EEG data acquisition (SET) analysis, can use the brain neurotransmitter $S$ spectrum corresponding to the system, such as -GABA (gamma aminobutyric Glu, prostaglandin D) (glutamic acid), 5-HT (5- Ach (HT), acetylcholine (NE), norepinephrine (DA), dopamine), excitatory neurotransmitter 3, inhibitory neurotransmitter 13, excitatory neurotransmitter 6.Respectively, in the brain of the brain around the frontal lobe, parietal lobe, occipital lobe and temporal lobe 16 electrode sampling, the reference electrode for the double measured ear.Shanghai Huicheng developed intelligent intelligent multi-functional decompression chamber (three generations of relaxation) HC-SX-ZNC model. Through the athlete's physiological sensor to collect the human body signal (heart rate, HRV, PNN50, respiratory rate, breathing ratio), assess the athletes are in the same level of relaxation of the state to vote pot practice, excluding the subjects of the inconsistency of the interference.

(2) Experimental Steps. Professor Yoga subjects abdominal breathing and chest breathing, the subjects easily grasp, then curling cast pot core technology essentials and practice yoga meditation and representation in yoga practice combining. Meditation and relaxation will link, curling a technology essentials become meditation and representation of lead to the athletes and the core technology of imagery training. And with the corresponding question and answer method, to test whether the subject has mastered the technology of casting pot, if not fully grasp, take prompt method, to remind them in the preparation of the pot when the appearance of the correct pot technology

The core of the test was conducted before and after the training in the Jilin Province Ice Center Athlete Lounge. In order to avoid interference during the experiment, each time a pilot athlete was experimented. The main test staff can skillfully control the operation of the entire experiment process, and can give the athletes to explain the entire experimental operation of the process and steps.

In the core technical representation training, we in order to make the experimental data measurement accuracy, reduce the error. Try to provide better environment and training conditions for curling athletes, we provide the subjects with professional and comfortable yoga mats. In the daily training (yoga balance exercise) process, require athletes to use chest breathing or abdominal breathing. In the two daily yoga training, a professional yoga instructor on the incorrect position of the athletes and breathing style is not correct for timely guidance to correct the athletes.

The subjects were informed the day before the experiment, asked to wash the hair before the test, reduce the head of the oil, to ensure the quality of the data and easy to collect. Requiring athletes to disable some paralysis of central drugs one or two days before the experiment, while not drinking alcohol and functional drinks, and to ensure sleep and its quality. In the collection process, try to let the athletes do not blink, do not speak and do not bite to reduce the EMG interference, while not allowed to wear watches, mobile phones and gold and silver jewelry and other things.

(3) Mathematical Statistics. All data were analyzed by SPSS13.0 statistical software for 2 (male and female) $\mathrm{x} 2$ (before and after the experiment) within or between groups. 


\section{Results and Analysis}

\section{The Results and Analysis of the Core Technical Appearance of the Curling Athletes Before and After the Experiment}

Table 1 Pot Stage of Core Technical of Curling Athletes in Imagery Training

\begin{tabular}{|c|c|c|c|c|c|c|c|c|c|c|c|c|}
\hline \multirow{2}{*}{$\begin{array}{l}\text { Gender } \\
\text { Male (pretest }\end{array}$} & \multicolumn{2}{|l|}{ S1 GABA } & \multicolumn{2}{|l|}{ S2 GA } & \multicolumn{2}{|c|}{ S3 Ach Receptors } & \multicolumn{2}{|l|}{ 5-HT } & \multicolumn{2}{|l|}{ NE } & \multicolumn{2}{|l|}{ DA } \\
\hline & \begin{tabular}{l}
\multicolumn{1}{c}{$\mathbf{M} \pm \mathbf{S}$} \\
$7.74 \pm 2.15$ \\
$13.32 \pm 7.77$
\end{tabular} & $\mathbf{F}$ & $\begin{array}{l}\mathbf{M} \pm \mathbf{S} \\
8.68 \pm 7.55 \\
10.32 \pm 2.34\end{array}$ & $\mathbf{F}$ & $\begin{array}{l}\mathbf{M} \pm \mathbf{S} \\
28.84 \pm 7.57 \\
20.63 \pm 13.13\end{array}$ & $\mathbf{F}$ & $\begin{array}{l}\mathbf{M} \pm \mathbf{S} \\
20.84 \pm 6.36 \\
17.05 \pm 4.66\end{array}$ & $\mathbf{F}$ & $\begin{array}{l}\mathbf{M} \pm \mathbf{S} \\
11.26 \pm 3.73 \\
15.89 \pm 4.53\end{array}$ & $\mathbf{F}$ & $\begin{array}{l}\mathbf{M} \pm \mathbf{S} \\
6.95 \pm 2.30 \\
5.53 \pm 2.09\end{array}$ & $\mathbf{F}$ \\
\hline Male(Rear) & & $8.59 * *$ & & $38.98^{* *}$ & & $5.88 *$ & & $4.99^{*}$ & & $10.99^{* *}$ & & 3.84 \\
\hline $\begin{array}{l}\text { Famale (pre } \\
\text { test) }\end{array}$ & $8.45 \pm 2.49$ & & $7.82 \pm 1.91$ & & $33.09 \pm 11.56$ & & $23.27 \pm 6.45$ & & $11.91 \pm 3.07$ & & $8.36 \pm 3.23$ & \\
\hline Famale (Rear) & $9.45 \pm 1.67$ & 1.73 & $10.55 \pm 2.35$ & 7. $84 * *$ & $29.55 \pm 7.01$ & 1.15 & $19.09 \pm 3.42$ & $5.64^{*}$ & $16.36 \pm 2.42$ & $12.30^{* *}$ & $5.64 \pm 1.87$ & $5.28 *$ \\
\hline
\end{tabular}

Note: *Represent $\mathrm{P}<0.05$, ${ }^{*}$ Represent $\mathrm{P}<0.01$ Because the icon is too large, there is no significant change in the indicators did not show

It can be seen from Table 1 that the curling athletes have significant differences in the preparation of the stage (S1), S1 (GABA), S2 (GA), S3 (Ach receptor), 5-HT, NE and DA.In the athletes ready to vote this stage, through their EEG alpha wave ultra-slow ups and downs of the data presented by the data analysis can be found in significant differences in the data, the male players were significantly higher than female players, Male and female players have different abilities. When the athlete performs the appearance, the potential at the left hemisphere of the brain changes. If the athlete's ability to level is relatively low, then the left side of his brain will change the ups and downs. It is because the athlete has the ability to appear slightly worse, so in the process of appearance, trying to use the self-cognitive ability, resulting in the left side of the brain potential fluctuations. The results also show that the more poorly performing players are, and there are some obstacles in the process of performing visual training, such as inconsistencies in the representation process, incomplete, or in a certain stage, followed by successive repetitions. When there will be some of my mind has nothing to do with the appearance of the content, so that athletes can not carry out the appearance of training. Male players than the female players like the effect of training is obviously because the boys in addition to training, in the usual will be active in some collective sports such as football, basketball and so on. In contrast, male athletes are more likely to have more athletes than female athletes. At present, there are many studies have proved that the use of scientific training methods, after a period of training after the performance will be improved, while the effect of good training, the ability to improve the performance of the players play in the game play a positive role The Because each athlete has a different ability to represent the different, so in the use of imaging training methods, to carry out this type of training for athletes to understand, and then for different athletes to design a scientific representation training methods. The same from the athletes on the training of such training on the degree of awareness of the training point of view, will directly affect the athletes in the training process is actively cooperate. If the athletes in the process of performing the training, the subjective willing to actively cooperate with the training effect will be more ideal, on the contrary, the athletes in the subjective manner on this training skeptical, then the effect of the appearance of training will produce a certain Impact. If the athletes get some improvement after the training, we can see that the athletes have been fully prepared at the initial stage of the game and have entered the game at the beginning of the pot. At this time, the athlete Will show a good competitive motive, the mind of the pot has a clear line and intensity control, psychological full of confidence. At the same time athletes gradually to enjoy the game, will be completely immersed in the game, which is the athlete's cerebral cortex nerve excitability gradually increased. And this degree of excitement is the most flexible. 
Different Age Curling Athletes in the Preparation of Potting Stage Before and After the Measurement of Training Neurotransmitters Changes

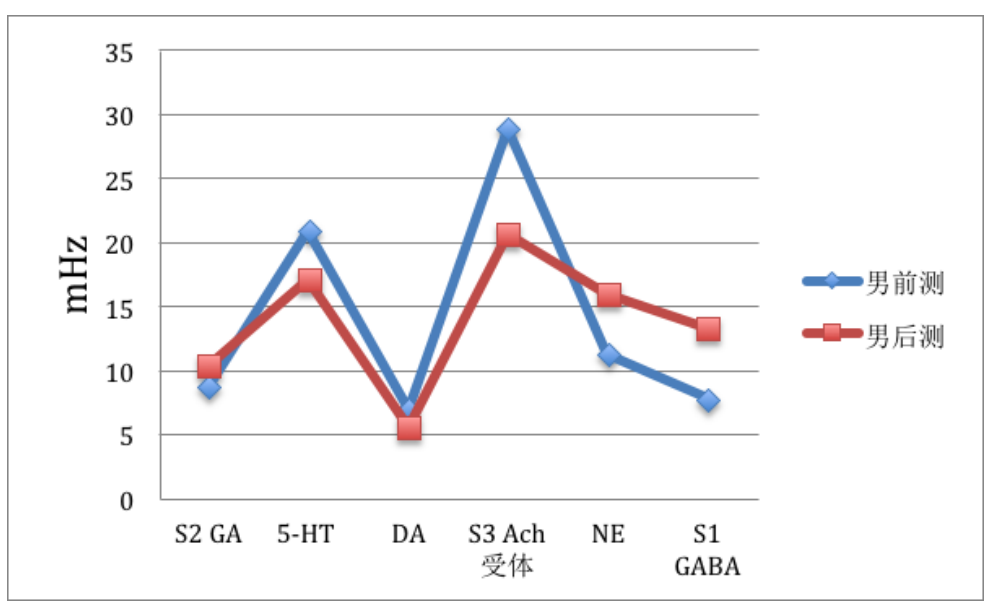

Figure 1. Male athlete imagery training for the pot stage before and after the change

It can be seen from Fig. 1, young men curling athletes in the appearance of training to prepare pot this stage before the test results can be measured before the S3 (Ach receptor) value of 29, after the measured value of 20, see the young men's athletes brain wave trend, Ups and downs change greatly. Through a six-month appearance training, in the post-test results, found that the potential of the brain waves tend to be stable.

Through the scientific representation training, athletes on the pot of the technical action of the attention point and the details of a new understanding, and in the brain of these information were re-integrated, so as to deepen the pot of technical action to understand and master, dynamic stereotypes And the feeling of muscle control force will also be more and more ideal. The expression of S3 (Ach receptor) acetylcholine receptor in the test results before and after EEG showed a decreasing trend. In participating in the brain excitement regulation S3 (Ach receptor) plays a major role. In the test before and after NE (norepinephrine) showed an upward trend, NE in the human brain is to promote the cortical excitability to reduce the role of inhibition of excitement. S3 (Ach receptor) acetylcholine receptors and NE (norepinephrine) are two neurotransmitters that primarily balance the excitatory and inhibitory effects of brain activity. In the daily training of athletes into a certain degree of scientific psychological training methods, help to improve their brain NE (norepinephrine) and S3 (Ach receptor) work ability. After a period of time the appearance of training, cast pot of this technical action in the details of the athletes in the brain can be presented one by one. Athletes in the appearance of training after recall, repeat and modify and re-create in the brain, it will form its own technical action. Athletes in the training efforts to make themselves feel the precise feeling of muscle force, enhance the accuracy of the technical action of the impression, and repeatedly strengthen the precise technical action, which can complete the most accurate power stereotypes. Therefore, the men's curling athletes after a period of training, brain waves in the S3 (Ach receptor) acetylcholine receptor showed a downward trend, NE (norepinephrine) showed an upward trend. Indicating that male curling athlete self-psychological control ability has been improved to overcome the possible negative emotions in the game. 


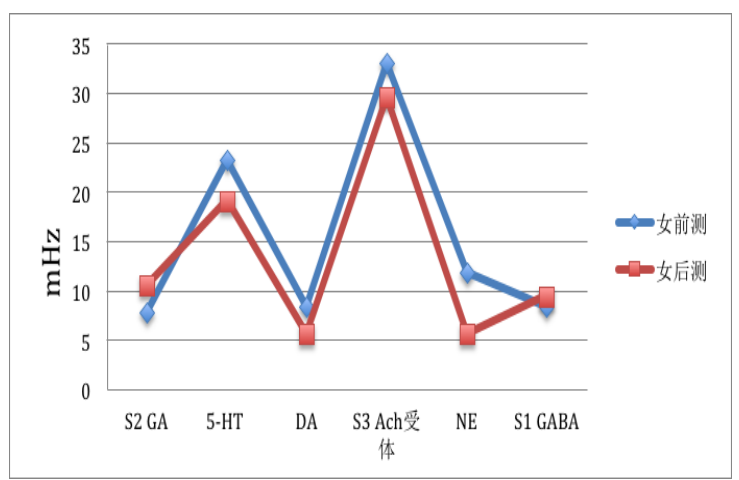

Figure 2. Female athlete imagery training for the pot stage before and after the change

It can be seen from Fig. 2, female curling athlete appearance training in the curling pot pot to prepare pot pot before and after the test, especially in the post-measured curve of the trend of S2GA (glutamic acid) after the test showed an upward trend, 5-HT (5 Serotonin), S3 (Ach receptor), NE (norepinephrine) and DA (dopamine) showed a decreasing trend.

Female curling athletes in the appearance of training before and after the test changes in the trend shows S2GA (glutamic acid) after the test showed an upward trend, indicating that female curling athletes after the appearance of training after attention has been raised in the preparation of the pot (Ach receptor) acetylcholine receptors showed a downward trend, which is conducive to inhibition of the brain is too excited; 5-HT (serotonin ) And NE (norepinephrine) showed a downward trend, indicating that the attention level of female curling players has been improved and improved accordingly, the anxiety of anxiety to a certain regulatory role; DA (dopamine) showed a decline Trend, dopamine in the human brain to play an important role in the regulation of exercise, mainly to regulate the body's muscle function, especially the degree of muscle tension, dopamine decline that female curling players muscle tension decreased. In the process of the female curling players throughout the pot, the technical action of natural skill, for the final curling stage to bring a better strength and angle. In the course of the game, the athlete curling shot speed is correct, shot strength is moderate, the shot angle is perfect for the final results of the game as a very important impact. Whether it is a little bit of change, the speed of change, or the angle of the deviation, will have a different impact on the trajectory of the curling, but also to the ice of the athletes to bring more trouble and difficult to make up for mistakes. At the same time curling Guoqing team members, most are in adolescence, girls in adolescence in the physical or psychological most of the early to the boys. And in this period of female athletes in terms of physical, or psychological will have a great change. So this at this stage, the female players will often show the emotional state of instability, particularly sensitive to certain things, when faced with even a very small problem will be impetuous, they are relatively male volleyball team members of the psychological activities to be more complex change. Through a period of appearance training, after the test results show that the mood of the women's athletes have been improved.

\section{Results and Recommendations}

Results. Different gender curling athletes ready to vote when the core technical appearance training, male and female athletes of the performance training test results for the $\mathrm{T}=1.97$ no significant difference. Male athletes in 5-HT (serotonin), Ach (acetylcholine), strong excitement, $\mathrm{NE}$ (norepinephrine), deep suppression, these indicators are slightly higher than girls.

There were significant differences in the levels of S1 (GABA), S2 (GA), S3 (Ach receptors), 5-HT, NE and DA in the preparation period.

The male and female athletes before and after image training EEG measured are S2GA (glutamic acid) were measured after the rise, S3 (Ach receptor), 5-HT (5- HT) and DA (dopamine) showing a downward trend. The ability to regulate emotions in the attention and through the representation training of sportsmen and women have been improved. The technology has been improved 


\section{Recommendations}

For the special psychological characteristics of curling athletes, in the daily curling training, the appropriate increase in the static balance of yoga training and yoga in the lead to the technical point of the guide, the best one week 3 times, each one and a half hours the most it is good.

In the training can also be appropriate to join the color therapy. Because color therapy is a relatively simple method, and effective faster, while no side effects. Color healing can lead to the most direct physical and psychological experience of athletes.

Integrate aromatherapy into training. Aromatherapy, in the subtle process, to help athletes improve the mood to enhance athletes self-confidence and so on. You can use essential oils in the dormitory and conference room, or use essential oils in the training and resting environment of the athlete. You can also apply essential oils to the ear and wrist of the athlete. Essential oils will slowly spread with the pulse of the beating, you can also produce essential oil sachets carry. Essential oil through the intake of breath and body absorption, in the process of psychological adjustment, pay attention to these usually bit by bit, in the subtle influence of improving the effect of psychological training.

The preparation of the curling exercise is an accurate exercise, and its concentration and mental state may be related to the characteristics of the brain waves with better athletic performance. It is expected to find the brain wave characteristics of the pot to improve the mental state before the action is performed, To achieve the peak sports results.

\section{Acknowledgements:}

Jilin Province Social Science Fund Project: Psychological Training of Chinese Young Curling Athletes and Its Innovation Path (2013BS86)

The first author: Lu Guo (1978-), male, Jilin, Jilin Agricultural University Department of military, associate professor, master, the main research direction for the physical education and training. Address: Changchun City, Jilin Province, Nan Guan Zone, 2888 New Town Avenue, Jilin Agricultural University Department of military teaching and research, Zip code: 130118. Tel: 13654399579. Email: 360282476@qq.com.

\section{References}

[1] Tan Weidong.Discussion on Several Problems in the Development of Chinese Curling Movement [J]. Ice and Snow Movement, 2003 (2): 53-55

[2] Behnn DG.Periodized Traning Program of the Canadian Olympic Curling Team[J]. National Strength Conditioning Association,2007.29(3):24-31

[3] World Curling Federation Secretariat.The Rules of Curling and Rules of Competition [M]. Perth:Olympic Winter Sport,2008,5-6

[4] Yu Liang, et al.Comparative Study on the Technical Characteristics of Chinese Women's Curling Team and European Team [J]. Journal of Sports Culture, 2010 (11): 65

[5] Wang Ke, Feng Wei. Technical analysis of curling pots [J]. Ice and Snow Sports, 2006 (2): $39-41$

[6] Wang Yu-feng et al.Study on the effectiveness of ice-washing in excellent Chinese curling athletes [J] .Harbin. Harbin Institute of Physical Education, 2015.6 (3): 36-40

[7] Yin Jiabao. Yin on the tennis game like memory changes of.[D] EEG and nerve transmitter in Jilin. Northeast Normal University master thesis, 2012:18-22

[8] Wang Fengchun. On the tactical awareness and training of curling athletes [J]. Ice and Snow Sports, 2012,34 (3): 48-51. 
[9] Zhang Shouzhong et al.. Specific cognitive ability China barrier characteristics of the women's curling four athletes J. Journal of Sports \& Science, 2012.20 (10): 27-28.

[10] Hongmei Ling. 2011 ice curling world championships affect the Chinese women won the analysis of factors [J]. Ice and Snow Sports, 33 (3): 36-38.

[11] Yang Yu, et al.Study on Special Psychological Ability of Elite Curling Athletes [J]. Ice and Snow Sports, 2011,33 (5): 45-50.

[12] Yu Xiao, etc. China's curling athletes psychological stress analysis and regulation [J]. Science and Education, 2011.

[13]Zhu Zhiqiang et al. Analysis of Scientific Research Status of Curling Movement [J]. Ice and Snow Sports, 2011,33 (5): 35-37.

[14] Ju Xiangwei et al.Discussion on the cognition and countermeasure of athlete 's psychological ability research [J]. Academic Discussion, 2010, (4): 47.

[15] Ma Yongjun, etc .. World women's curling team technical strength analysis [J]. Ice and Snow Sports, 2010,32 (4): 37-40.

[16]Zhang Wei et al. Psychological characteristics and regulation of juvenile curling athletes [J]. Ice and Snow Sports, 2010,32 (3): 65-66.

[17]Zhang Zhipeng et al. The origin of the curling movement and its current development [J]. Journal of Harbin Institute of Physical Education, 2010,28 (5): 26-29.

[18] Robert S. Weinberg, Daniel Gould. Foundations of Sport Exercise Psychology [M]. Edwards Brothers, 2002: 77-79.

[19] Bob weeks. Curling for dummies[M].CDG Books Canada Inc. 2001: 188 -190

[20] Marshall B. All internal training. all the time. Fraced coach,1996(3):31-36.

[21] KleiueD.Anxiety and sport performance analysis[J].anxiety Research, 1990 (2):60-65. 